\title{
Extracellular Vesicles Release from Human Osteoarthritic Chondrocytes
}

\author{
Ilaria Giusti ${ }^{1}$, Marianna Di Francesco ${ }^{1}$, Annalisa Mancò ${ }^{2}$, Sandra D’Ascenzo $^{1}$, Antonello Aloisi ${ }^{2}$, Vittorio \\ Calvisi ${ }^{2}$ and Vincenza Dolo ${ }^{1 *}$ \\ ${ }^{1}$ Department of Life, Health and Environmental Sciences, University of L'Aquila, Italy \\ ${ }^{2}$ Department of Life, Health and Environmental Sciences, Postgraduate School of Orthopaedics and Traumatology, University of L'Aquila, Italy
}

Submission: February 14, 2017; Published: March 02, 2017

*Corresponding author: Vincenza Dolo, Department of Life, Health and Environmental Sciences, University of L’Aquila, Via Vetoio-Coppito 2 , I-67100, L'Aquila, Italy, Tel: +39 0862 433665; Fax: +39 0862 433425; Email: vincenza.dolo@univaq.it

\begin{abstract}
Osteoarthritis (OA) is a degenerative disease which affects up to $40 \%$ of people aged over 65 years causing joint pain, stiffness/impaired mobility and having a significant negative effect on their quality of life. It is beginning to emerge that EVs could have a role in this disease pathogenesis: indeed, over the years the role of EVs as a mean of cell-to-cell communication has become increasingly clear and obvious; specifically, in $\mathrm{OA}$ it seems that chondrocytes and synoviocytes, the two most important cell types in joint biology, affect each other their behavior using EVs to mediate the exchange of information. In spite of this, the study of EVs released from these cells is very limited. Here we present a morphological analysis of EVs isolated from OA chondrocytes, showing both their release from cell surface (using Scansion Electron Microscopy) and their size and morphology (using Transmission Electron Microscopy); moreover, being known the role of enzymes in the degradation of the extracellular matrix associated to $\mathrm{OA}$, we assessed the presence of Gelatinases and Plasminogen Activators associated to EVs.
\end{abstract}

\section{Introduction}

In recent years it has become more and more clear that both prokaryotic and eukaryotic cells are able to release, in extracellular medium, spherical membrane vesicles delimited by a lipid bilayer of various size and cell origin [1]. In relation to the latter two parameters currently is it possible to distinguish between: exosomes (EXOs) which originate from the endosomal compartment after fusion of multivesicular bodies with the plasma membrane and whose size is typically considered smaller than $100 \mathrm{~nm}$ in diameter; microvesicles (MVs) which are released by outward budding of cell membrane and have diameter between 100 and $1000 \mathrm{~nm}$; apoptotic bodies (ABs) which are the larger vesicles, having a diameter greater than $1 \mu \mathrm{m}$, and are released by cells undergoing apoptosis [2].

EVs, initially interpreted as cell debris or simply as a mechanism that cell used to discard useless components, are now believed to be a previously not recognized form of intercellular communication since they contain fully functional proteins, lipids and nucleic acids which travel protected between releasing and target cells, even in very distant places from the source site [1]; they, indeed, could be isolated from virtually all biological fluids included blood, saliva, urine, pleural effusions, ocular effluent and aqueous humor, nasal secretions, breast milk, amniotic fluid, cerebrospinal fluid, bile, semen and ascites [3].

The undeniable role in inter-cellular communication make EVs involved in many normal and pathological conditions: some examples of physiological functions include immune system functions [4], hemostasis and thrombosis [5], interneuronal communication [6], male [7] and female reproduction [8] whilst pathological processes mainly include cancer [9-11] but also malignancies of nervous system [12], rheumatoid arthritis and osteoarthitis [13]. The present paper briefly discloses the release of EVs (both MVs and EXOs) by human chondrocytes isolated from osteoarthritic joints.

\section{Materials and Methods}

\section{Cell culture}

Specimens of human OA articular cartilage were obtained from femoral cut (femoral condyles and intercondylar notch) of patients undergoing total knee replacement surgery. Written informed consent was obtained from all participants. For cells isolation cartilage was removed from the subcondral bone using a sterile sharp scalpel and placed in serum free DMEM (Dulbecco's Modified Eagle's Medium - High Glucose; EuroClone 
S.p.A., Pero (MI), Italy); once all cartilage was removed, slivers were further diced to improve the subsequent enzyme action. Enzymatic isolation was carried out by an overnight digestion in $1 \mathrm{mg} / \mathrm{ml}$ Collagenase II (Collagenase Type II from Clostridium histolyticum; Gibco-ThermoFisher, Waltham, Massachusetts, USA) in DMEM supplemented with 10\% heat-inactivated Fetal Bovine Serum (FBS - EuroClone).

After digestion, cell suspension was filtered on cell strainers (Falcon $^{\mathrm{TM}}$ Cell Strainers, mesh size $70 \mu \mathrm{m}$; FisherScientific, ThermoFisher) to remove undigested debris and centrifuged (600 xg, 10 minutes, room temperature). Cells were then resuspended and plated in DMEM containing 1x Penicillin/ Streptomycin (Euroclone SpA), $2 \mathrm{mM}$ L-Glutamine (Euroclone SpA), 10 mM Hepes (Sigma-Aldrich, St. Louis, MO, USA), 2,5 $\mu \mathrm{g} /$ $\mathrm{ml}$ Amphotericin B (Sigma-Aldrich) and 10\% FBS.

\section{Isolation of EVs from conditioned medium}

Human OA chondrocytes were stimulated with $5 \%$ of $40 \mathrm{~nm}$ filtered FBS HyClone (Thermo Scientific, Rockford, IL, USA) in DMEM for 18 hours and then conditioned medium was collected. To isolate EVs, the conditioned medium obtained as above was centrifuged at $600 \mathrm{xg}$ for $15 \mathrm{~min}$ and then at 1,500 x g for 30 min to remove cells and large debris, respectively. Supernatants were centrifuged at $100,000 \mathrm{x}$ g for $2 \mathrm{~h}$ at $4^{\circ} \mathrm{C}$ in a Beckmann ultracentrifuge. Isolated vesicles were resuspended in Dulbecco's phosphate-buffered saline (EuroClone, Milan, Italy) for TEM analysis and protein content analysis. Double determination of vesicle quantification was carried out by measuring the vesicleassociated protein levels using the Bradford method (Bio-Rad, Milan, Italy) with bovine serum albumin (Sigma-Aldrich, St. Louis, MO, USA) as the standard.

\section{Electron microscopy}

Scanning electron microscopy (SEM) was performed on cells that were allowed to attach directly on coverslips, simultaneously to their isolation step. When cells were almost $70 \%$ confluent, samples were fixed with $2 \%$ glutaraldehyde (Electron Microscopy Sciences, Hatfield, PA, USA) in phosphatebuffered saline (PBS) for 30 minutes, then dehydrated by subsequent exchanges of the following ethanol gradual series: $30,50,70,90$, and $100 \%$ ethanol diluted in distilled water. Samples were critical point-dried, glued onto stubs, coated with gold in a SCD040 Balzer Sputterer, and observed using a Philips $505 \mathrm{SEM}$ at $20 \mathrm{kV}$.

Transmission electron microscopy (TEM) was carried out on isolated EVs, resuspended in PBS, to analyze their ultrastructural morphology. According to proper dilutions, the samples were transferred on 300 mesh carbon-coated copper grids (Electron Microscopy Sciences, Hatfield, PA, USA) in a humidified chamber at room temperature and allowed to adsorb to the surface of the grids for 5 minutes. EVs on grids were then fixed in $2 \%$ glutaraldehyde (Electron Microscopy Sciences, Hatfield, PA, USA) in PBS for 10 minutes and then briefly rinsed in Milli- $Q$ water. The grids were kept wet on the side of the adsorbed EVs during all steps, but dry on the opposite side. To contrast the samples, grids were transferred on a drop of $2 \%$ phosphotungstic acid, brought to $\mathrm{pH} 7.0$ with $\mathrm{NaOH}$, for 5 minutes. After negative staining the grids were left to dry and stored in appropriate grid storage boxes. Grids with adhered EVs were examined with a Philips CM 100 transmission electron microscope TEM at $80 \mathrm{kV}$ and images were captured by a Kodak digital camera.

\section{Zymography}

Gelatin and casein-plasminogen zymography were performed on EVs released from OA chondrocytes.

Gelatin zymography was performed using sodium dodecyl sulfate-polyacrylamide gels (SDS-PAGE, 7.5\%) copolymerized with $1 \mathrm{mg} / \mathrm{mL}$ of gelatin type B (Sigma Chemical Co.) whilst casein-plasminogen zymography was performed using SDSPAGE 10\% copolymerized with 0,2\% casein (Sigma Aldrich, St. Louis, MO, USA) and $10 \mathrm{mg} / \mathrm{ml}$ plasminogen (Sigma Aldrich, St. Louis, MO, USA). EVs were diluted in an SDS-PAGE sample buffer in nonreducing conditions without heating; $10 \mu \mathrm{g}$ and $15 \mu \mathrm{g}$ of EVs were loaded for gelatin and casein-plasminogen zymography, respectively.

After electrophoresis, the gels were washed twice for 30 minutes in Tris $50 \mathrm{mM} \mathrm{pH} 7.4$ plus $2.5 \%$ Triton X-100 at RT and incubated overnight in activating buffer $(50 \mathrm{mM}$ Tris- $\mathrm{HCl}, \mathrm{pH}$ 7.4, containing $5 \mathrm{mM} \mathrm{CaCl} 2$ and $150 \mathrm{mM} \mathrm{NaCl}$ for gelatinases; Tris $50 \mathrm{mM} \mathrm{pH} 7.4$ for plasminogen activators) at $37^{\circ} \mathrm{C}$. The gels were stained with Coomassie Blue R 250 (Bio-Rad, Hercules, CA) using a mixture of methanol-acetic acid-water (4:1:5) for 1 hour and were destained in the same solution without dye. The enzymes' activities were visualized as distinct bands, indicating the proteolysis of the substrate. Images recording and bands intensity quantification was carried out with the gel documentation system Alliance LD2 (UVItec, Cambridge, UK).

\section{Results and Discussion}

Osteoarthritis (OA) is a degenerative disease which affects the synovial joints; whole articulation, i.e. hyaline cartilage and subchondral bone, is involved. The pathology, in fact, is characterized by cartilage degradation, synovial inflammation and alteration of bone structure [13-15].

OA has a slow evolution, characterized by joint pain, stiffness/ impaired mobility and by significant negative effects on the quality of life. It is certainly the most common musculoskeletal disorder and cause of chronic pain in the elderly: up to $40 \%$ of people aged over 65 years typically has symptomatic OA of the knee or hip [14,16].

Currently, research in OA field is aimed at identify new approaches to stimulate the repair of damaged cartilage [14]; so, a deeper understanding of the biology of the cells involved, 
mainly represented by chondrocytes, could be helpful to this purpose.

The release of EVs from chondrocytes is not well documented, as well as their possible role in OA [13], even if it has been demonstrated that EVs are someway involved in the pathology acting as means of communication between synoviocytes and chondrocytes: EVs from chondrocytes treated with IL-1 $\beta$ when used to treat synoviocytes induced an increased production of MMP-13, which leads progression of OA [17] as well as EVs from fibroblast-like synoviocytes stimulated with IL-1 $\beta$ induce osteoarthritic changes in chondrocytes (up regulation of MMP13 and down regulation of collagen type 2 and aggrecan genes) [18]. To our best knowledge no morphological studies on EVs from OA chondrocytes have never been performed and this paper intends to fill this gap in this topic.

To such purpose, we used electron microscopy to study the release of EVs by OA Chondrocytes. The presence of microvesicles on the surface of the cell membrane was identified only in some cells, where considerable dynamisms of the cell membrane together with the shedding of microvesicles occurred in the central region of the plasma membrane and not on the whole cell surface (Figure 1). In particular, TEM analyses of isolated EVs displayed intact vesicles enclosed by the plasma membrane (Figures 2A-2E) and characterized by heterogeneous size, ranging from $52 \mathrm{~nm}$ to $707 \mathrm{~nm}$ (Figure 2F), confirming the release of both EXOs and MVs by OA chondrocytes. Sometimes, we could identify EVs interspersed in collagen fibers, co-pelletted with EVs at the ultracentrifugation step (Figure 2E).

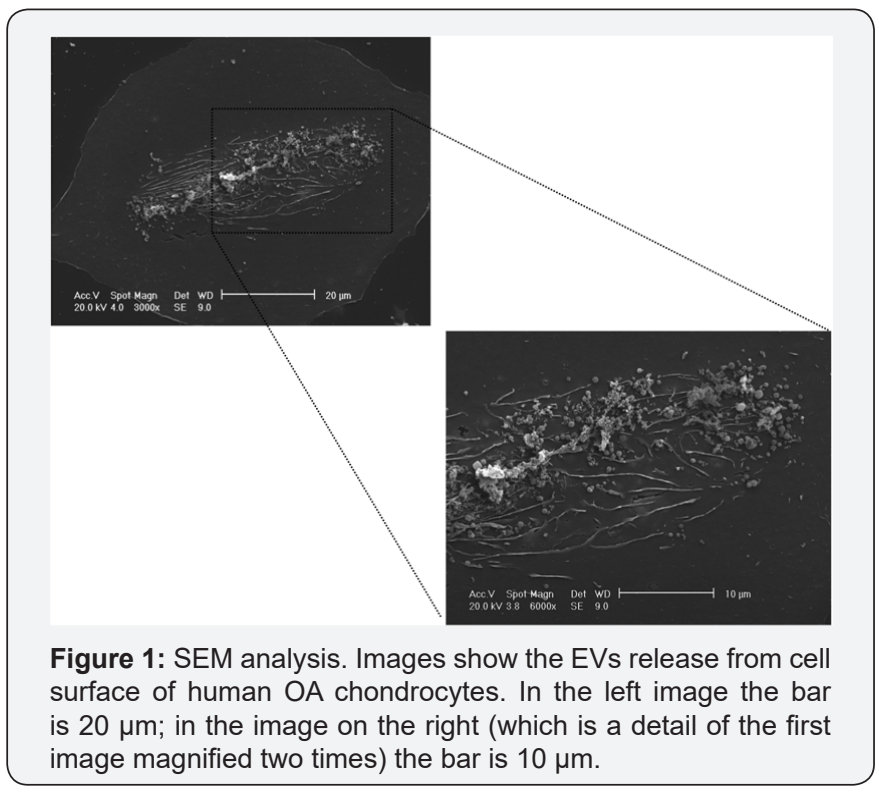

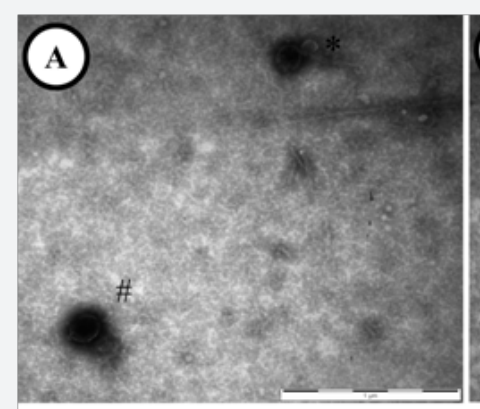
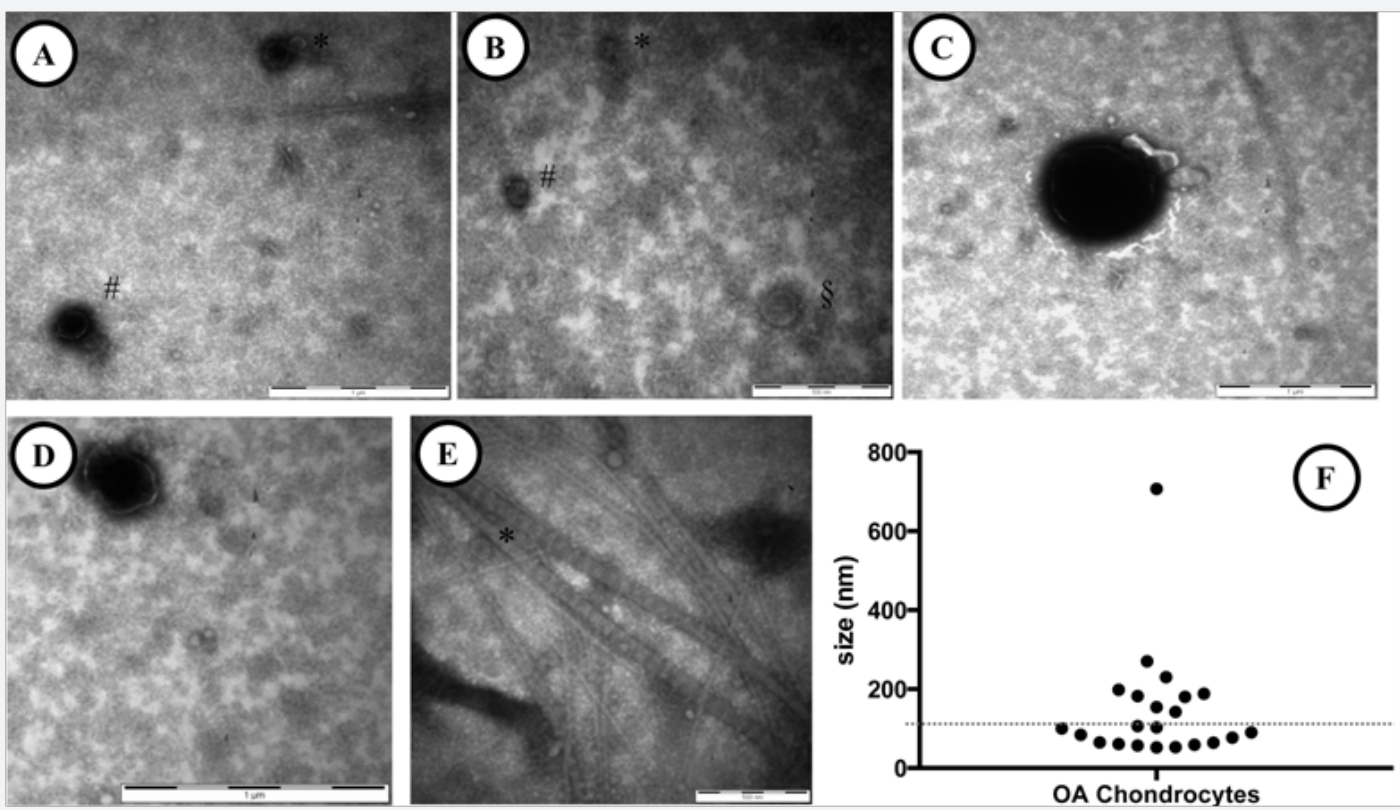

Figure 2: TEM analysis. Transmission electron microscopy images of vesicles isolated from conditioned medium of human OA chondrocytes. (A) Bar $1 \mu \mathrm{m}$ (EVs size: *154nm, \# 188nm); (B) Bar 500nm (EVs size: * $52 \mathrm{~nm}$, \# $65 \mathrm{~nm}, \S 130 \mathrm{~nm}$ ); (C) Bar $1 \mu \mathrm{m}$ (EV size 707nm); (D) Bar $1 \mu \mathrm{m}$ (EV size 270nm); (E) Image shows an EV and many collagen fibers. Bar 500nm (EV size 110nm); (F) Graph shows the size of EVs analyzed from transmission electron microscopy images: each EV observed on the grids corresponds to one dot. The horizontal bar is the size limit $(100 \mathrm{~nm})$ that usually distinguishes exosomes and microvesicles subpopulations.

Moreover, it is known that in the pathogenesis of $\mathrm{OA}$ both the chondrocytes and the extracellular matrix (ECM) are fundamental to maintain healthy articular cartilage [13]. Healthy ECM balance is primarily maintained by chondrocytes who produce the main component of matrix: collagen type II and the proteoglycan aggrecan [19]. OA is characterized by the unbalance between synthesis and breakdown of the ECM; the latter is believed to be largely due to MMPs action, in particular MMP-13. [20].

So, we proceeded to verify if other enzyme activity were associated to OA chondrocytes-derived EVs, assuming that they 
also could have a possible role in the breakdown of the matrix. Zymography assays showed the presence of both gelatinases and plasminogen activators (PAs) (Figure 3). Many lytic bands were evidenced by gelatinase zymography; some of them probably correspond to complexes of gelatinases (100-119 kDa) whilst the others are easily recognized as MMP-9 (88 kDa), pro-MMP-2 (74 kDa) and MMP-2 (63 kDa). Similarly, several lytic bands were present in casein-plasminogen assay probably ascribable to PAI (Plasminogen Activators Inhibitors)-PA complexes (92 $\mathrm{kDa}$ ), tissue-type Plasminogen (66 kDa) and urokinase-type Plasminogen (44,7-49 kDa).

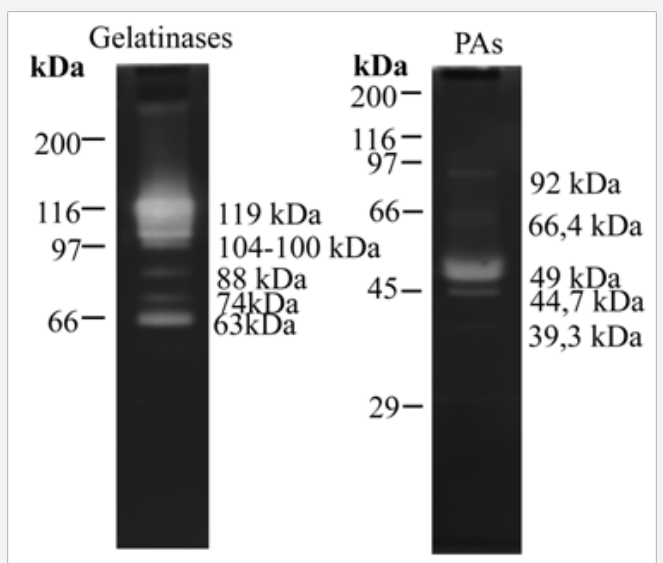

Figure 3: Zymography assays. Gelatinases (left image) and plasminogen activators (PAs) (right image) were analyzed by means of gelatin and casein-plasminogen zymography, respec $\neg$ tively. Standard molecular weights are exhibited on the left of each image. Calculated molecular weight for each lytic band is exhibited on the right of each image.

\section{Conclusion}

In conclusion, the data reported in this paper prove that the release of EVs actually occurs in OA chondrocytes; the analysis of their size shows that we have a mixed population consisting of both MVs and EXOs. In addition, to EVs are associated enzymatic activities that may have a role in disease progression.

\section{Acknowledgment}

This work was partially supported by Fondazione Cassa di Risparmio della Provincia dell'Aquila.

\section{References}

1. Maas SL, Breakefield XO, Weaver AM (2016) Extracellular Vesicles: Unique Intercellular Delivery Vehicles. Trends Cell Biol, pii: S09628924(16): 30179-9.

2. György B, Szabó TG, Pásztói M, Pál Z, Misják P, et al. (2011) Membrane vesicles, current state-of-the-art: emerging role of extracellular vesicles. Cell Mol Life Sci 68(16): 2667-2688.
3. Witwer KW, Buzás EI, Bemis LT, Bora A, Lässer C, et al. (2013) Standardization of sample collection, isolation and analysis methods in extracellular vesicle research. J Extracell Vesicles 27: 2.

4. Robbins PD, Morelli AE (2014) Regulation of immune responses by extracellular vesicles. Nat Rev Immunol 14(3): 195-208.

5. Owens AP, Mackman N (2011) Microparticles in hemostasis and thrombosis. Circ Res 108(10): 1284-1297.

6. Chivet M, Javalet C, Hemming F, Pernet-Gallay K, Laulagnier K, et al. (2013) Exosomes as a novel way of interneuronal communication. Biochem Soc Trans 41(1): 241-244.

7. Aalberts M, Stout TA, Stoorvogel W (2013) Prostasomes: extracellular vesicles from the prostate. Reproduction 147(1): R1-14.

8. Nardi Fda S, Michelon TF, Neumann J, Manvailer LF, Wagner B, et al. (2016) High levels of circulating extracellular vesicles with altered expression and function during pregnancy. Immunobiology 221(7): 753-760.

9. Fujita Y, Yoshioka, Ochiya T (2016) Extracellular vesicle transfer of cancer pathogenic components. Cancer Sci 107(4): 385-390.

10. Giusti I, Dolo V (2014) Extracellular vesicles in prostate cancer: new future clinical strategies? Biomed Res Int 2014: 561-571

11. Naito Y, Yoshioka Y, Yamamoto Y, Ochiya T (2016) How cancer cells dictate their microenvironment: present roles of extracellular vesicles. Cell Mol Life Sci 74(4): 697-713.

12. Rajendran L, Honsho M, Zahn TR, Keller P, Geiger KD, et al. (2006) Alzheimer's disease beta-amyloid peptides are released in association with exosomes. Proc Natl Acad Sci USA 103(30): 11172-11177.

13. Withrow J, Murphy C, Liu Y, Hunter M, Fulzele S, et al. (2016) Extracellular vesicles in the pathogenesis of rheumatoid arthritis and osteoarthritis. Arthritis Res Ther 18(1): 286.

14. Di Sante L, Villani C, Santilli V, Valeo M, Bologna E, et al. (2016) Intraarticular hyaluronic acid vs platelet-rich plasma in the treatment of hip osteoarthritis. Med Ultrason 18(4): 463-468.

15. Dallari D, Stagni C, Rani N, Sabbioni G, Pelotti P, et al. (2016) Ultrasound-guided injection of Platelet-Rich Plasma and Hyaluronic Acid, separately and in combination, for hip osteoarthritis: a randomized controlled study. Am J Sports Med 44(3): 664-671.

16. Mlynarek RA, Kuhn AW, Bedi A (2016) Platelet-Rich Plasma (PRP) in Orthopedic Sports Medicine. Am J Orthop (Belle Mead NJ) 45(5): 290-326.

17. Nakasa T, Miyaki S, Kato T, Takada T, Nakamura Y, et al. (2012) Exosome derived from osteoarthritis cartilage induces catabolic factor gene expressions in synovium. ORS 2016 Annual Meeting, San Francisco, USA, Trans Orth Res Soc 2012: Abstract 708.

18. Kato T, Miyaki S, Ishitobi H, Nakamura Y, Nakasa T, et al. (2014) Exosomes from IL-1beta stimulated synovial fibroblasts induce osteoarthritic changes in articular chondrocytes. Arthritis Res Ther 16(4): R163.

19. Thirion S, Berenbaum F (2004) Culture and phenotyping of chondrocytes in primary culture. Methods Mol Med 100: 1-14.

20. Fosang AJ, Last K, Knauper V, Murphy G, Neame PJ (1996) Degradation of cartilage aggrecan by collagenase-3 (MMP-13). FEBS Lett 380(1-2): 17-20. 
(C) Commons Attribution 4.0 License

BY DOI: 10.19080/IJCSMB.2017.01.555572
Your next submission with Juniper Publishers will reach you the below assets

- Quality Editorial service

- Swift Peer Review

- Reprints availability

- E-prints Service

- Manuscript Podcast for convenient understanding

- Global attainment for your research

- Manuscript accessibility in different formats

( Pdf, E-pub, Full Text, Audio)

- Unceasing customer service

Track the below URL for one-step submission https://juniperpublishers.com/online-submission.php 